\title{
A triply cubic polynomials approach for globally convergent algorithm in coefficient inverse problems
}

\author{
Quan-Fang Wang \\ Mechanical and Automation Engineering, \\ The Chinese University of Hong Kong, Shatin, N. T., Hong Kong \\ E-mail: quanf angwang@hotmail.com, qf wang@mae.cuhk.edu.hk
}

\begin{abstract}
In this paper, a triply cubic polynomials approach is proposed firstly for solving the globally convergent algorithm of coefficient inverse problems in three dimension. Using Taylor type expansion for three arguments to construct tripled cubic polynomials for the approximate solution as identifying coefficient function of parabolic initial-boundary problems, in which unknown coefficients appeared at nonlinear term. The presented computational approach is effective to execute in spatial 3D issues arising in real world. Further, the complete algorithm can be straightforwardly performed to lower or higher dimensions case.
\end{abstract}

\section{Background}

Worldwidelly, current BQP (Bi-quadratic polynomials) approach is used to solve the globally convergent algorithm of coefficient inverse problems in the spatial dimension of two. Since it had been proposed in $[1,2]$, recognized and named as BQP approach in article [3], fortunately, it already had been adopted in numerous publications such as [4], [5], [6, 7, 8, 9], and so forth. Actually, the methodology is worked to recover the profile of realistic systems (parabolic, hyperbolic, elliptic type) in industrial, electricmagnetic, optical frequency sounding, engineer, medical tomography, physical as well as chemical fields. The question arising in here is for high dimension cases: what suitable approach for three dimension case? whether it possess enough smoothness to simply use BQP approach in three dimension? whether there is exact approach to construct the approximate solution in coefficients inverse problems of 3D or high dimensions? To find the answer, in this paper, we introduce the perfect methodology to make the completeness of globally convergent algorithm in three dimension case. Our idea is using Taylor type expansion for three arguments to construct tripled cubic polynomials for the approximate solution in finding coefficient of parabolic initial-boundary problems, in which unknown coefficient appeared at nonlinear term. Indeed, one can use the provided 
triplet cubical polynomials in the sequential minimization algorithm in convexification (convex performance index) approach, it leads to get a stable approximate solution via minimizing finite sequences of strictly convex objective function. Through the posed triple cubic polynomials approach, the determination of unknown coefficient can be effectively solved for a broad class applications, which was restricted in $1 \mathrm{D} / 2 \mathrm{D}$ case at [10], [11] and [12]. In addition, recent years reports on adaptive technique (cf. $[13,14,15,16])$ are indirect method with its limitation.

The purpose of the article is to address the triple cubic polynomials approach for solving coefficient inverse problems in three dimension at the globally convergent algorithm, upon with previous bi-quadratic polynomials methodology given in [3] for two dimension case. The systematical theoretical proposals and computational procedure are feasible in 3-dimensional case. For verifying, numerical demonstration and laboratory experiments are desired as evidences and complementaries in our future works.

We divided this paper into 5 sections. Section 2 is to set the mathematical formulation of the coefficient inverse problem for nonlinear parabolic initial-boundary equation. Section 3 is to propose the triply cubic polynomials approach for globally convergent algorithm associated with parabolic coefficient inverse problems in three dimension case. In Section 4, the convergence and error estimate are proved for the proposed approach and criteria function. Section 5 is to supply the computational procedure for numerical experiments. Lastly, Section 6 contains the conclusions.

\section{Preliminaries}

In order to interpret the newly triple cubic polynomials approach, firstly, it needs to give the mathematical setting for nonlinear parabolic initial-boundary problems with unknown coefficient appeared in nonlinearity. Consider the Cauchy problem described in the form of nonlinear parabolic partial differential equation at $\mathbf{R}^{3} \times(0, \infty)$ for $(\mathbf{x}, t)$, $\mathbf{x}=\left(x_{1}, x_{2}, x_{3}\right)$

$$
\left\{\begin{array}{l}
\frac{\partial u(\mathbf{x}, t)}{\partial t}-\nabla^{2} u(\mathbf{x}, t)+k(\mathbf{x}) u(\mathbf{x}, t)=-s(\mathbf{x}, t), \\
\left.\frac{\partial u(\mathbf{x}, t)}{\partial \eta}\right|_{t=0}+\left.a u(\mathbf{x}, t)\right|_{t=0}=u_{0}(\mathbf{x})
\end{array}\right.
$$

where $k(\mathbf{x}) \geq 0$ is an unknown coefficient (usually, it is considered as $k(\mathbf{x}, \mathbf{u})$ without lost of generality and nonlinearity. In this paper, we take $k(\mathbf{x})$. To stress the new approach, the measurement and noise of $k$ is neglected at setting the issue), $a$ is a constant and

$\frac{\partial}{\partial \eta}$ represents the unit outer normal derivative in the Robin initial condition. Let

$$
\mathbf{R}_{+}^{3}=\left\{\mathbf{x} \in \mathbf{R}^{3} \mid x_{1}>0 \text { and } x_{2}>0 \text { and } x_{3}>0\right\}
$$


and $S$ denotes the plane $S=\left\{\left(x_{2}, x_{3}\right) \mid x_{1}=0\right\}$. Assume that the source function $s(\mathbf{x}, t)=\delta\left(\mathbf{x}-\mathbf{x}_{0}\right) \delta(t) \geq 0$ is compactly supported in $\mathbf{R}^{3}$. Let $\Omega \subset \mathbf{R}_{+}^{3}$ be a cubic domain defined by

$$
\Omega=\left\{\mathbf{x} \in \mathbf{R}_{+}^{3} \mid x_{1} \in(0, L), x_{2} \in(0, L), x_{3} \in(0, L)\right\} .
$$

The support of $s(\mathbf{x}, t)$ belongs to the set $\mathbf{R}_{+}^{3} \backslash \Omega$. Using the convexification approach (cf. [11]) to denote the Laplace transform of $u(\mathbf{x}, t)$ by

$$
w(\mathbf{x}, s)=\int_{0}^{\infty} e^{-s t} u(\mathbf{x}, t) d t
$$

where the parameter $s>0$. Then applying Laplace transform to the Cauchy problem (2.1) to obtain

$$
\left\{\begin{array}{l}
{[s+k(\mathbf{x})] w-\nabla^{2} w=S_{0}(\mathbf{x}, s),} \\
\lim _{|\mathbf{x}| \rightarrow \infty} w=0, \\
\frac{\partial w(\mathbf{x}, s)}{\partial \eta}+a w(\mathbf{x}, s)=w_{0}(\mathbf{x}, s), \quad \mathbf{x} \in S .
\end{array}\right.
$$

In here, $\frac{\partial w}{\partial \eta}$ is the outer normal derivative of $w$ at the boundary. The $w_{0}(\mathbf{x}, s)$ and $S_{0}(\mathbf{x}, s)$ denote Laplace transforms of $u_{0}$, and the source function $s(x, t)$, respectively. That is

$$
\begin{aligned}
& w_{0}(\mathbf{x}, 0)=\mathcal{L}\left(u_{0}\right)=\int_{0}^{\infty} e^{-s t} u_{0}(\mathbf{x}) d t \\
& S_{0}(\mathbf{x}, s)=\mathcal{L}(s(\mathbf{x}, t))=\int_{0}^{\infty} e^{-s t} \delta\left(\mathbf{x}-\mathbf{x}_{0}\right) \delta(t) d t=\delta\left(\mathbf{x}-\mathbf{x}_{0}\right),
\end{aligned}
$$

where $\mathcal{L}$ represents the Laplace transform operator. For simplicity, assume that $S_{0}(\mathbf{x}, s)=\delta\left(\mathbf{x}-\mathbf{x}_{0}\right)$, where $\mathbf{x}_{0}=\left(0, x_{2}, L-l_{0}\right) \in \mathbf{R}_{+}^{3} \backslash \Omega$ is a fixed source position, and $l_{0}$ is a small positive number. For the positivity of the function $w(\mathbf{x}, s)$, refer Lemma 1 and Lemma 2 of [11].

Definition 2.1. For the system (2.1), given data $s(\mathbf{x}, t)$ on $w(\mathbf{x}, s), \mathbf{x} \in S$ for a fixed source position $\mathbf{x}_{0}$ and sufficiently large $T$. The coefficient inverse problem (CIP) is to identify the unknown coefficient $k(\mathbf{x})$ in domain $\Omega$.

Remark 2.2. Definition 2.1 provides the existence and uniqueness of solution $u(\mathbf{x}, t)$. For unknown coefficients $k(\mathbf{x})$, it provide the existence but it can not guarantee the uniqueness.

Next, we intend to deduce equivalent equation without the unknown coefficient $k(\mathbf{x})$ from the nonlinear parabolic equation (2.1). Cite [3] for two dimension case, and refer Lemma 2 of [11] for 1D case. Actually, whether the source function $S_{0}(\mathbf{x}, t)$ exist or not, it is convenient to neglect this term in following deduces. One can complement 
this term if necessary, the dealing is appeared in papers $[10,11,12,13,14,15,16]$. For $w>0$ in $\Omega$, set $v=\ln w$ and transform (2.2) as

$$
\nabla^{2} v+(\nabla v)^{2}=s+k(\mathbf{x}), \quad \mathbf{x} \in \Omega .
$$

For the explanation of transformation method (see [3] for different selection), if introducing

$$
f(\mathbf{x}, s)=v, \quad q(\mathbf{x}, s)=\frac{\partial f}{\partial s}
$$

then the formulation (2.3) and Lemma 2 of [11] implies that

$$
f(\mathbf{x}, s)=O\left(s^{1}\right), \quad q(\mathbf{x}, s)=O(1) \text { as } s \rightarrow \infty .
$$

The integral on $(s, \infty)$ can be approximated as

$$
f(\mathbf{x}, s) \approx-\int_{s}^{\bar{s}} q(\mathbf{x}, \nu) d \nu
$$

where $\bar{s}$ is a sufficiently large number (cf. [11]) for getting resultant value. Take $s>s_{0}>0$, for $\mathbf{x} \in \Omega, \Gamma=\partial \Omega$ and $s \in\left(s_{0}, \bar{s}\right)$, using the integral on corresponding time variable $\nu$, and substituting (2.4) to (2.3), the boundary problem (2.2) equivalent to the following nonlinear integro-differential system

$$
\left\{\begin{array}{l}
\int_{s}^{\bar{s}} \nabla^{2} q(\mathbf{x}, \nu) d \nu+\left(\int_{s}^{\bar{s}} \nabla q(\mathbf{x}, \nu) d \nu\right)^{2}-s=0 \\
\frac{\partial q(\mathbf{x}, s)}{\partial \eta}+a q(\mathbf{x}, s)=q_{0}(\mathbf{x}, s), \quad \mathbf{x} \in \Gamma
\end{array}\right.
$$

In here, $q_{0}$ correspond to $w_{0}$. Obviously, $\mathbf{R}_{+}^{3}$ is open set, the normal derivative of (2.5) is valid on the entire domain. The boundary condition is transformed from (2.2), the following is details.

$$
\begin{array}{ll}
\text { Set } \frac{\partial v}{\partial \eta}=\frac{\partial(\ln w)}{\partial \eta} ; v=\ln w ; v_{0}=\ln w_{0}, & \text { then } \frac{\partial v}{\partial \eta}+a v=v_{0} . \\
\text { Set } f(\mathbf{x}, s)=v, & \text { then } \frac{\partial f(\mathbf{x}, s)}{\partial \eta}+a f(\mathbf{x}, s)=f_{0}(\mathbf{x}, s) . \\
\text { Set } \frac{\partial}{\partial \eta}\left(\frac{\partial f}{\partial s}\right) ; q(\mathbf{x}, s)=\frac{\partial f}{\partial s} ; q_{0}(\mathbf{x}, s)=\frac{\partial f_{0}}{\partial s}, & \text { then } \frac{\partial q(\mathbf{x}, s)}{\partial \eta}+a q(\mathbf{x}, s)=q_{0}(\mathbf{x}, s) .
\end{array}
$$

For numerical computation, $q_{0}$ can be calculated by using regularizing scheme of numerical differential on $s$. Above integral make the $k(\mathbf{x})$ disappeared, resultantly, (2.5) is the expected system without containing the unknown coefficient $k(\mathbf{x})$. Define the following set of functions

$$
G\left(n^{\prime}\right)=\left\{q(\mathbf{x}, s) \mid \frac{\partial q}{\partial \eta}+a q=q_{0}, \mathbf{x} \in \Gamma, \max _{s \in\left[s_{0}, \bar{s}\right]}\|q(\mathbf{x}, s)\|_{C^{3}(\Omega)} \leq n^{\prime}\right\},
$$

where $n^{\prime}>0$ is a given number. 
Theorem 2.3. There exists at most one solution $q(\mathbf{x}, s)$ to problem (2.5) such that $\max _{s \in\left[s_{0}, \bar{s}\right]}\|q(\mathbf{x}, s)\|_{C^{3}(\Omega)}<\infty$.

The proof of Theorem 2.3 can be obtained using the result of [11].

\section{Triply cubic polynomials approach}

\subsection{Integro-differential vectors system}

Suppose a regular domain $\Omega=[0, L] \times[0, L] \times[0, L]$ for $L>0$. Divide $\Omega$ by the partition of $N=n \times n \times n$ cubic lattices with $n$ number intervals at each direction of $x_{i}, i=1,2,3$. Set $\Omega_{1}=\left\{\left(x_{1}, x_{2}, x_{3}\right) \mid-\mathbf{R} \leq x_{1}, x_{2}, x_{3} \leq \mathbf{R}\right\}$ for fixed real number $\mathbf{R}>0$. Assume that $q_{0}$ is observed on $\Gamma=\partial \Omega_{1} \approx \Omega \cap S$. Take $\left.\left\{\phi_{k}\left(x_{1}, x_{2}, x_{3}\right)\right\}\right|_{n=1} ^{\mathbf{n}} \subset C^{3}\left(\bar{\Omega}_{1}\right)$ as a set of cubic basis functions that approximate $q(\mathbf{x}, s)$ and its $\mathbf{x}$-derivatives up to the third order, i.e.,

$$
D_{\mathbf{x}}^{\alpha} q(\mathbf{x}, s) \approx D_{\mathbf{x}}^{\alpha} \sum_{n=1}^{\mathbf{n}} \eta_{n}(s) \phi_{n}\left(x_{1}, x_{2}, x_{3}\right), \quad(\mathbf{x}, s) \in \Omega \times\left[s_{0}, \bar{s}\right],|\alpha| \leq 3,
$$

where $\eta_{n}(s), n=1,2, \ldots, \mathbf{n}$ is the expansion coefficients depended on $s$. Then from Theorem 2.3, for $q \in G\left(n^{\prime}\right)$, there is a number $\mathbf{n}\left(\varepsilon, n^{\prime}\right)$ can be chosen corresponding to $\varepsilon, n^{\prime}$ for sufficiently small $\varepsilon>0$ and $\eta_{k}(s)$, such that

$$
\max _{s \in\left(s_{0}, \bar{s}\right)}\left\|q(\mathbf{x}, s)-\sum_{n=1}^{\mathbf{n}\left(\varepsilon, n^{\prime}\right)} \eta_{n}(s) \phi_{n}\left(x_{1}, x_{2}, x_{3}\right)\right\|_{C^{3}(\Omega)}<\varepsilon .
$$

With the results in [3] on two dimension parabolic coefficient inverse problems for space variables $\left(x_{2}, x_{3}\right)$ and time variable $s$, suppose

$$
q(\mathbf{x}, s) \approx \sum_{n=1}^{\mathbf{n}} \eta_{n}(s) \phi_{n}\left(x_{1}, x_{2}, x_{3}\right), \quad(\mathbf{x}, s) \in \Omega \times\left[s_{0}, \bar{s}\right] .
$$

Then, one can deduced that

$$
\nabla^{2} q(\mathbf{x}, s)=\sum_{n=1}^{\mathbf{n}} \eta_{n}(s) \frac{\partial^{2} \phi_{n}\left(x_{1}, x_{2}, x_{3}\right)}{\partial x_{1}^{2}}+\sum_{n=1}^{\mathbf{n}} \eta_{n}(s) \frac{\partial^{2} \phi_{n}\left(x_{1}, x_{2}, x_{3}\right)}{\partial x_{2}^{2}}+\sum_{n=1}^{\mathbf{n}} \eta_{n}(s) \frac{\partial^{2} \phi_{n}\left(x_{1}, x_{2}, x_{3}\right)}{\partial x_{3}^{2}} .
$$

That is $\nabla^{2} q(\mathbf{x}, s)=\sum_{n=1}^{\mathbf{n}} \eta_{n}(s) \nabla^{2} \phi_{n}\left(x_{1}, x_{2}, x_{3}\right)$. Substituting (3.1) into (2.5), therefore, the initial-boundary parabolic problem (2.5) imply that

$$
\left\{\begin{array}{l}
\int_{s}^{\bar{s}} \sum_{n=1}^{\mathbf{n}} \eta_{n}(\nu) \nabla^{2} \phi_{n}\left(x_{1}, x_{2}, x_{3}\right) d \nu+\left(\int_{s}^{\bar{s}} \sum_{n=1}^{\mathbf{n}} \eta_{n}(\nu) \nabla \phi_{n}\left(x_{1}, x_{2}, x_{3}\right) d \nu\right)^{2}-s=0, \\
\sum_{n=1}^{\mathbf{n}} \eta_{n}(s) \frac{\partial \phi_{n}\left(x_{1}, x_{2}, x_{3}\right)}{\partial \eta}+a \sum_{n=1}^{\mathbf{n}} \eta_{n}(s) \phi_{n}\left(x_{1}, x_{2}, x_{3}\right)=\sum_{n=1}^{\mathbf{n}} \eta_{n}^{0}(s) \phi_{n}\left(x_{1}, x_{2}, x_{3}\right),
\end{array}\right.
$$


where

$$
q_{0}(\mathbf{x}, s) \approx \sum_{n=1}^{\mathbf{n}} \eta_{n}^{0}(s) \phi_{n}\left(x_{1}, x_{2}, x_{3}\right)
$$

is the expansion of boundary value $q_{0}(\mathbf{x}, s)$ according to the basis functions $\left\{\phi_{n}\left(x_{1}, x_{2}, x_{3}\right)\right\}$ on boundary point $\mathbf{x}=\left(x_{1}, x_{2}, x_{3}\right) \in \Gamma$, and $\eta_{n}^{0}(s)$ are their expansion coefficients for $n=1,2, \ldots, \mathbf{n}$. Using the vector form to denote that

$$
\mathbf{p}\left(x_{1}, x_{2}, x_{3}, s\right)=\left(\begin{array}{c}
\eta_{1}(s) \phi_{1}\left(x_{1}, x_{2}, x_{3}\right) \\
\vdots \\
\eta_{n}(s) \phi_{n}\left(x_{1}, x_{2}, x_{3}\right) \\
\vdots \\
\eta_{\mathbf{n}}(s) \phi_{\mathbf{n}}\left(x_{1}, x_{2}, x_{3}\right)
\end{array}\right) .
$$

For clearly, the detailed first and second order derivatives of $\mathbf{p}\left(x_{1}, x_{2}, x_{3}, s\right)$ are given by

$$
\begin{aligned}
& \mathbf{p}_{1}^{\prime}=\frac{\partial \mathbf{p}}{\partial x_{1}}=\left(\frac{\eta_{1}(s) \partial \phi_{1}\left(x_{1}, x_{2}, x_{3}\right)}{\partial x_{1}}, \cdots \frac{\eta_{n}(s) \partial \phi_{n}\left(x_{1}, x_{2}, x_{3}\right)}{\partial x_{1}}, \cdots \frac{\eta_{\mathbf{n}}(s) \partial \phi_{\mathbf{n}}\left(x_{1}, x_{2}, x_{3}\right)}{\partial x_{1}}\right)^{T}, \\
& \mathbf{p}_{2}^{\prime}=\frac{\partial \mathbf{p}}{\partial x_{2}}=\left(\frac{\eta_{1}(s) \partial \phi_{1}\left(x_{1}, x_{2}, x_{3}\right)}{\partial x_{2}}, \cdots \frac{\eta_{n}(s) \partial \phi_{n}\left(x_{1}, x_{2}, x_{3}\right)}{\partial x_{2}}, \cdots \frac{\eta_{\mathbf{n}}(s) \partial \phi_{\mathbf{n}}\left(x_{1}, x_{2}, x_{3}\right)}{\partial x_{2}}\right)^{T}, \\
& \mathbf{p}_{3}^{\prime}=\frac{\partial \mathbf{p}}{\partial x_{3}}=\left(\frac{\eta_{1}(s) \partial \phi_{1}\left(x_{1}, x_{2}, x_{3}\right)}{\partial x_{3}}, \cdots \frac{\eta_{n}(s) \partial \phi_{n}\left(x_{1}, x_{2}, x_{3}\right)}{\partial x_{3}}, \cdots \frac{\eta_{\mathbf{n}}(s) \partial \phi_{\mathbf{n}}\left(x_{1}, x_{2}, x_{3}\right)}{\partial x_{3}}\right)^{T}, \\
& \mathbf{p}_{1}^{\prime \prime}=\frac{\partial^{2} \mathbf{p}}{\partial x_{1}^{2}}=\left(\frac{\eta_{1}(s) \partial^{2} \phi_{n}\left(x_{1}, x_{2}, x_{3}\right)}{\partial x_{1}^{2}}, \cdots \frac{\eta_{n}(s) \partial^{2} \phi_{n}\left(x_{1}, x_{2}, x_{3}\right)}{\partial x_{1}^{2}}, \cdots \frac{\eta_{\mathbf{n}}(s) \partial^{2} \phi_{\mathbf{n}}\left(x_{1}, x_{2}, x_{3}\right)}{\partial x_{1}^{2}}\right)^{T}, \\
& \mathbf{p}_{2}^{\prime \prime}=\frac{\partial^{2} \mathbf{p}}{\partial x_{2}^{2}}=\left(\frac{\eta_{1}(s) \partial^{2} \phi_{n}\left(x_{1}, x_{2}, x_{3}\right)}{\partial x_{2}^{2}}, \cdots \frac{\eta_{n}(s) \partial^{2} \phi_{n}\left(x_{1}, x_{2}, x_{3}\right)}{\partial x_{2}^{2}}, \cdots \frac{\eta_{\mathbf{n}}(s) \partial^{2} \phi_{\mathbf{n}}\left(x_{1}, x_{2}, x_{3}\right)}{\partial x_{2}^{2}}\right)^{T}, \\
& \mathbf{p}_{3}^{\prime \prime}=\frac{\partial^{2} \mathbf{p}}{\partial x_{3}^{2}}=\left(\frac{\eta_{1}(s) \partial^{2} \phi_{n}\left(x_{1}, x_{2}, x_{3}\right)}{\partial x_{3}^{2}}, \cdots \frac{\eta_{n}(s) \partial^{2} \phi_{n}\left(x_{1}, x_{2}, x_{3}\right)}{\partial x_{3}^{2}}, \cdots \frac{\eta_{\mathbf{n}}(s) \partial^{2} \phi_{\mathbf{n}}\left(x_{1}, x_{2}, x_{3}\right)}{\partial x_{3}^{2}}\right)^{T} .
\end{aligned}
$$

Use the notations $\mathbf{p}^{\prime}=\left(\mathbf{p}_{1}^{\prime}, \mathbf{p}_{2}^{\prime}, \mathbf{p}_{3}^{\prime}\right), \mathbf{p}^{\prime \prime}=\left(\mathbf{p}_{1}^{\prime \prime}, \mathbf{p}_{2}^{\prime \prime}, \mathbf{p}_{3}^{\prime \prime}\right)$ if necessary. For unified framework of the parabolic initial-boundary problem, roughly set the integro-differential operator $\mathbf{L}$ expressed as

$$
\mathbf{L}(\mathbf{p})=F\left(\int_{s}^{\bar{s}} \mathbf{p}^{\prime \prime}\left(x_{1}, x_{2}, x_{3}, \nu\right) d \nu, \int_{s}^{\bar{s}} \mathbf{p}^{\prime}\left(x_{1}, x_{2}, x_{3}, \nu\right) d \nu, x, y, z, s\right) .
$$

Resultantly, from (3.2) and (3.4) to yield that

$$
\left\{\begin{array}{l}
\mathbf{L}(\mathbf{p})=0, \mathbf{p}=\mathbf{p}\left(x_{1}, x_{2}, x_{3}, s\right), x_{1} \in(0, L), x_{2} \in(0, L), x_{3} \in(0, L), s \in\left(s_{0}, \bar{s}\right), \\
\frac{\partial \mathbf{p}\left(x_{1}, x_{2}, x_{3}, s\right)}{\partial \eta}+a \mathbf{p}\left(x_{1}, x_{2}, x_{3}, s\right)=\mathbf{p}_{0}\left(x_{1}, x_{2}, x_{3}, s\right), \quad\left(x_{1}, x_{2}, x_{3}\right) \in \Gamma
\end{array}\right.
$$


In (3.5), $\mathbf{p}_{0}$ is a vector corresponding to the initial value $q_{0}$ in (2.5). In details, that is

$$
\mathbf{p}_{0}\left(x_{1}, x_{2}, x_{3}, s\right)=\left(\begin{array}{c}
\eta_{1}^{0}(s) \phi_{1}\left(x_{1}, x_{2}, x_{3}\right) \\
\vdots \\
\eta_{n}^{0}(s) \phi_{n}\left(x_{1}, x_{2}, x_{3}\right) \\
\vdots \\
\eta_{\mathbf{n}}^{0}(s) \phi_{\mathbf{n}}\left(x_{1}, x_{2}, x_{3}\right)
\end{array}\right)
$$

where $\eta_{n}^{0}(s), n=1,2, \ldots, \mathbf{n}$ are coefficients of $q_{0}$ given in (3.3).

\subsection{Extrapolated boundary condition}

Eventually, the third kind boundary condition in (2.1) can be converted to Dirichlet boundary condition, In here, we omit the redundancies for the limitation of paper length. In short, it is instructed for three dimension case initially.

Let $G \subset \mathbf{R}^{3}$ be a cube large than domain $\Omega=[0, L] \times[0, L] \times[0, L], \Omega \subset G$, and 0 be the center of $G$. Select such a $G$, for any point $\mathbf{x} \in G$ which not near a corner, there exists a unique point $\mathbf{x}^{\prime} \in \partial \Omega$ such that the outward distance in normal direction is given by $\left|\mathbf{x}-\mathbf{x}^{\prime}\right|=a_{0} l_{t}$ for $a_{0}>0$. Then $\partial G$ is an extrapolated boundary of 3D domain $\Omega$. Using the extrapolated boundary in nonlinear initial-boundary parabolic system $(2.1)$ of $u(\mathbf{x}, t)$ to get

$$
\frac{\partial u}{\partial \eta}(\mathbf{x}, t)=\lim _{\alpha \rightarrow 0} \frac{u(\mathbf{x}+\alpha \eta, t)-u(\mathbf{x}, t)}{\alpha} .
$$

Since $\mathbf{x}+a_{0} l_{t} \eta \in \partial G$, it implies $u\left(\mathbf{x}+a_{0} l_{t} \eta, t\right)=0$. If the number $a_{0} l_{t}$ is sufficiently-small, then $\frac{\partial u}{\partial \eta}(\mathbf{x}, t) \approx-\frac{1}{a_{0} l_{t}} u(\mathbf{x}, t)$. Assume that

$$
\frac{\partial u}{\partial \eta}(\mathbf{x}, t)=-\frac{1}{a_{0} l_{t}} u(\mathbf{x}, t), \mathbf{x} \in \partial \Omega, t \in(0, T) .
$$

In the case of the extrapolated boundary condition $\left.\frac{\partial u}{\partial \eta}\right|_{\partial \Omega} \equiv 0$, then condition (3.6) is equivalent to the third kind boundary condition $\frac{\partial u(\mathbf{x}, t)}{\partial \eta}+a u(\mathbf{x}, t)=0$ on $\partial \Omega$ for $t \in(0, T)$, where $a=1 /\left(a_{0} l_{t}\right)$. That is, using (3.6), the original third kind boundary condition is convert to Dirichlet boundary condition $\left.u(\mathbf{x}, t)\right|_{t=0}=-a_{0} l_{t} u_{0}(\mathbf{x})$ for $\mathbf{x} \in \partial \Omega$.

\subsection{Triply cubic polynomials}

To propose the triply cubic approach in the construction of approximate solution, discretise the coefficient inverse problems (3.2) on the cubic domain $\Omega=[0, L] \times$ 
$[0, L] \times[0, L]$. Individually, set the cubical partition lattices with the coordinate in $x=x_{1}, y=x_{2}$ and $z=x_{3}$ direction as

$$
\begin{array}{ll}
0=x_{0}<x_{1}<\ldots<x_{i-1}<x_{i}<\ldots<x_{N}=L, & h^{i}=x_{i}-x_{i-1}, \quad i=1,2, \ldots, N . \\
0=y_{0}<y_{1}<\ldots<y_{j-1}<y_{j}<\ldots<y_{N}=L, & h^{j}=y_{j}-y_{j-1}, \quad j=1,2, \ldots, N . \\
0=z_{0}<z_{1}<\ldots<z_{k-1}<z_{k}<\ldots<z_{N}=L, & h^{k}=z_{k}-z_{k-1}, \quad k=1,2, \ldots, N .
\end{array}
$$

In here, $h^{i}, h^{j}, h^{k}$ are the partition interval lengths at each direction $x, y$ and $z$, respectively. For domain $\Omega$, suppose that the triply cubic polynomial at each lattice is a vector $\mathbf{p}_{i j k}(x, y, z, s)$, their indices are $j=1,2, \ldots, N, j=1,2, \ldots, N$ and $k=1,2, \ldots, N$. Its detail is

$$
\mathbf{p}_{i j k}(x, y, z, s)=\left(p_{i j k}^{1}(x, y, z, s), \cdots, p_{i j k}^{n}(x, y, z, s), \cdots, p_{i j k}^{\mathbf{n}}(x, y, z, s)\right)
$$

for $\mathbf{n}=N^{3}$. Without confusion, each cubic lattice is denoted as $C_{i j k}$ corresponding polynomial vector $\mathbf{p}_{i j k}$. Specially, the cubic lattices with triply cubic polynomials numbers are plotted in Figure 1.

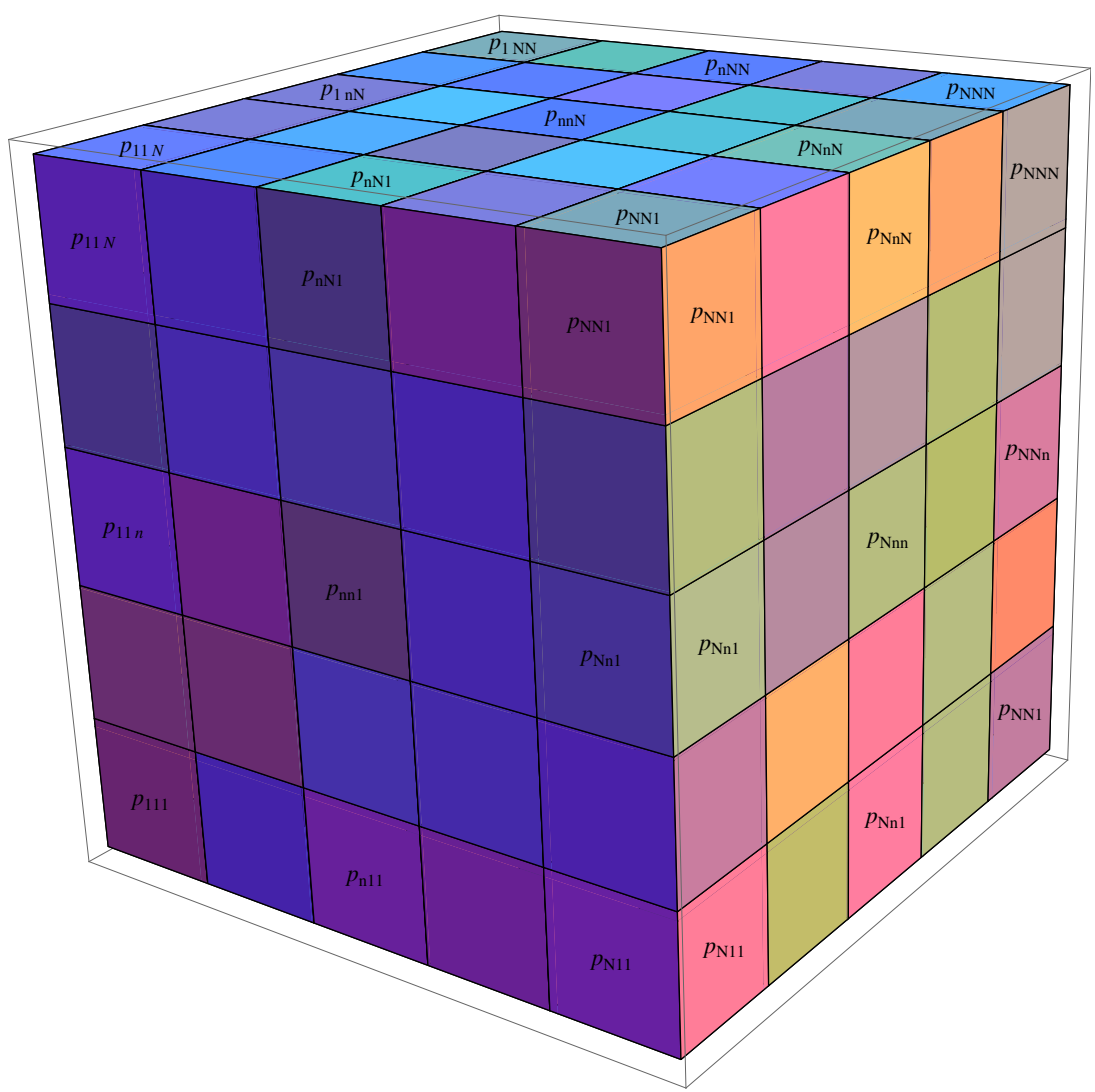

Figure 1. Cubic lattices with corresponding triply cubic polynomials (the structure is analogical a colorful cubical magic square)

We list its front (blue), above (cyan) and right (pink) numbers in Table 1, Table 2, Table 3, and omit others inside cubic lattices numbers. 
Table 1. Front (blue) grids of cubic lattices in Figure 1.

\begin{tabular}{|c|c|c|c|c|}
\hline$p_{11 N}$ & $\cdots$ & $p_{n N 1}$ & $\cdots$ & $p_{N N 1}$ \\
\hline$\vdots$ & & $\vdots$ & & $\vdots$ \\
\hline$p_{11 n}$ & $\cdots$ & $p_{n n 1}$ & $\cdots$ & $p_{N n 1}$ \\
\hline$\vdots$ & & $\vdots$ & & $\vdots$ \\
\hline$p_{111}$ & $\cdots$ & $p_{n 11}$ & $\cdots$ & $p_{N 11}$ \\
\hline
\end{tabular}

Table 2. Above (cyan) grids of cubic lattices in Figure 1.

\begin{tabular}{|c|c|c|c|c|}
\hline$p_{1 N N}$ & $\cdots$ & $p_{n N N}$ & $\cdots$ & $p_{N N N}$ \\
\hline$\vdots$ & & $\vdots$ & & $\vdots$ \\
\hline$p_{1 n N}$ & $\cdots$ & $p_{n n N}$ & $\cdots$ & $p_{N n N}$ \\
\hline$\vdots$ & & $\vdots$ & & $\vdots$ \\
\hline$p_{11 N}$ & $\cdots$ & $p_{n N 1}$ & $\cdots$ & $p_{N N 1}$ \\
\hline
\end{tabular}

Table 3. Right (pink) grids of cubic lattices in Figure 1.

\begin{tabular}{|c|c|c|c|c|}
\hline$p_{N N 1}$ & $\cdots$ & $p_{N n N}$ & $\cdots$ & $p_{N N N}$ \\
\hline$\vdots$ & & $\vdots$ & & $\vdots$ \\
\hline$p_{N n 1}$ & $\cdots$ & $p_{N n n}$ & $\cdots$ & $p_{N N n}$ \\
\hline$\vdots$ & & $\vdots$ & & $\vdots$ \\
\hline$p_{N 11}$ & $\cdots$ & $p_{N n 1}$ & $\cdots$ & $p_{N N 1}$ \\
\hline
\end{tabular}

The idea of this paper is to construct the approximate solution with the Taylor type expansion of 3 variables. Using a Taylor type expansion for three arguments to a function $f^{T}(x, y, z, s)$ (upper index ' $\mathrm{T}$ ' represents 'Taylor expansion') at point $\left(x_{0}, y_{0}, z_{0}, s\right)$. Since the original Taylor expansion contains 64 terms, and the leading term $\left(x-x_{0}\right)^{3}\left(y-y_{0}\right)^{3}\left(z-z_{0}\right)^{3}$ is 9 order polynomial. In order to reduce the complexity of computation, a Taylor type expansion is used in our calculations with 20 terms, and 
its leading terms $\left(x-x_{0}\right)^{3},\left(y-y_{0}\right)^{3},\left(z-z_{0}\right)^{3}$, and $\left(x-x_{0}\right)\left(y-y_{0}\right)\left(z-z_{0}\right)$ are 3 order polynomials. The expanded polynomial in detail is in the form of

$$
\begin{aligned}
& f^{T}(x, y, z, s) \\
= & \frac{\partial^{3} f^{T}\left(x_{0}, y_{0}, z_{0}, s\right)}{\partial x^{3}} \frac{\left(x-x_{0}\right)^{3}}{3 !}+\frac{\partial^{3} f^{T}\left(x_{0}, y_{0}, z_{0}, s\right)}{\partial y^{3}} \frac{\left(y-y_{0}\right)^{3}}{3 !}+\frac{\partial^{3} f^{T}\left(x_{0}, y_{0}, z_{0}, s\right)}{\partial z^{3}} \frac{\left(z-z_{0}\right)^{3}}{3 !} \\
& +3 \frac{\partial^{3} f^{T}\left(x_{0}, y_{0}, z_{0}, s\right)}{\partial x^{2} \partial y} \frac{\left(x-x_{0}\right)^{2}}{2 !}\left(y-y_{0}\right)+3 \frac{\partial^{3} f^{T}\left(x_{0}, y_{0}, z_{0}, s\right)}{\partial x \partial y^{2}}\left(x-x_{0}\right) \frac{\left(y-y_{0}\right)^{2}}{2 !} \\
& +3 \frac{\partial^{3} f^{T}\left(x_{0}, y_{0}, z_{0}, s\right)}{\partial x^{2} \partial z} \frac{\left(x-x_{0}\right)^{2}}{2 !}\left(z-z_{0}\right)+3 \frac{\partial^{3} f^{T}\left(x_{0}, y_{0}, z_{0}, s\right)}{\partial y^{2} \partial z} \frac{\left(y-y_{0}\right)^{2}}{2 !}\left(z-z_{0}\right) \\
& +3 \frac{\partial^{3} f^{T}\left(x_{0}, y_{0}, z_{0}, s\right)}{\partial x \partial z^{2}}\left(x-x_{0}\right) \frac{\left(z-z_{0}\right)^{2}}{2 !}+3 \frac{\partial^{3} f^{T}\left(x_{0}, y_{0}, z_{0}, s\right)}{\partial y \partial z^{2}}\left(y-y_{0}\right) \frac{\left(z-z_{0}\right)^{2}}{2 !} \\
& +6 \frac{\partial^{3} f^{T}\left(x_{0}, y_{0}, z_{0}, s\right)}{\partial x \partial y \partial z}\left(x-x_{0}\right)\left(y-y_{0}\right)\left(z-z_{0}\right) \\
& +\frac{\partial^{2} f^{T}\left(x_{0}, y_{0}, z_{0}, s\right)}{\partial x^{2}} \frac{\left(x-x_{0}\right)^{2}}{2 !}+\frac{\partial^{2} f^{T}\left(x_{0}, y_{0}, z_{0}, s\right)}{\partial y^{2}} \frac{\left(y-y_{0}\right)^{2}}{2 !} \\
& +\frac{\partial^{2} f^{T}\left(x_{0}, y_{0}, z_{0}, s\right)}{\partial z^{2}} \frac{\left(z-z_{0}\right)^{2}}{2 !}+2 \frac{\partial^{2} f^{T}\left(x_{0}, y_{0}, z_{0}, s\right)}{\partial x \partial y}\left(x-x_{0}\right)\left(y-y_{0}\right) \\
& +2 \frac{\partial^{2} f^{T}\left(x_{0}, y_{0}, z_{0}, s\right)}{\partial x \partial z}\left(x-x_{0}\right)\left(z-z_{0}\right)+2 \frac{\partial^{2} f^{T}\left(x_{0}, y_{0}, z_{0}, s\right)}{\partial y \partial z}\left(y-y_{0}\right)\left(z-z_{0}\right) \\
& +\frac{\partial f^{T}\left(x_{0}, y_{0}, z_{0}, s\right)}{\partial x}\left(x-x_{0}\right)+\frac{\partial f^{T}\left(x_{0}, y_{0}, z_{0}, s\right)}{\partial y}\left(y-y_{0}\right) \\
& +\frac{\partial f^{T}\left(x_{0}, y_{0}, z_{0}, s\right)}{\partial y}\left(z-z_{0}\right)+f^{T}\left(x_{0}, y_{0}, z_{0}, s\right) .
\end{aligned}
$$

Re-denote each coefficient functions of the expanded Taylor type polynomial (3.7) as

$$
\begin{aligned}
& a_{1}(s)=\frac{\partial f^{T}\left(x_{0}, y_{0}, z_{0}, s\right)}{\partial x}, a_{2}(s)=\frac{\partial f^{T}\left(x_{0}, y_{0}, z_{0}, s\right)}{\partial y}, a_{3}(s)=\frac{\partial f^{T}\left(x_{0}, y_{0}, z_{0}, s\right)}{\partial z}, \\
& a_{11}(s)=\frac{\partial^{2} f^{T}\left(x_{0}, y_{0}, z_{0}, s\right)}{\partial x^{2}}, a_{22}(s)=\frac{\partial^{2} f^{T}\left(x_{0}, y_{0}, z_{0}, s\right)}{\partial y^{2}}, a_{33}(s)=\frac{\partial^{2} f^{T}\left(x_{0}, y_{0}, z_{0}, s\right)}{\partial z^{2}}, \\
& a_{12}(s)=\frac{\partial^{2} f^{T}\left(x_{0}, y_{0}, z_{0}, s\right)}{\partial x \partial y}, a_{23}(s)=\frac{\partial^{2} f^{T}\left(x_{0}, y_{0}, z_{0}, s\right)}{\partial y \partial z}, a_{13}(s)=\frac{\partial^{2} f^{T}\left(x_{0}, y_{0}, z_{0}, s\right)}{\partial x \partial z}, \\
& a_{111}(s)=\frac{\partial^{3} f^{T}\left(x_{0}, y_{0}, z_{0}, s\right)}{\partial x^{3}}, a_{222}(s)=\frac{\partial^{3} f^{T}\left(x_{0}, y_{0}, z_{0}, s\right)}{\partial y^{3}}, a_{333}(s)=\frac{\partial^{3} f^{T}\left(x_{0}, y_{0}, z_{0}, s\right)}{\partial z^{3}}, \\
& a_{112}(s)=\frac{\partial^{3} f^{T}\left(x_{0}, y_{0}, z_{0}, s\right)}{\partial x^{2} \partial y}, a_{122}(s)=\frac{\partial^{3} f^{T}\left(x_{0}, y_{0}, z_{0}, s\right)}{\partial x \partial z^{2}}, a_{113}(s)=\frac{\partial^{3} f^{T}\left(x_{0}, y_{0}, z_{0}, s\right)}{\partial x^{2} \partial z} \\
& a_{223}(s)=\frac{\partial^{3} f^{T}\left(x_{0}, y_{0}, z_{0}, s\right)}{\partial y^{2} \partial z}, a_{133}(s)=\frac{\partial^{3} f^{T}\left(x_{0}, y_{0}, z_{0}, s\right)}{\partial x \partial z^{2}}, a_{233}(s)=\frac{\partial^{3} f^{T}\left(x_{0}, y_{0}, z_{0}, s\right)}{\partial y \partial z^{2}}, \\
& a_{123}(s)=\frac{\partial^{3} f^{T}\left(x_{0}, y_{0}, z_{0}, s\right)}{\partial x \partial y \partial z} .
\end{aligned}
$$


Therefore, using (3.8), the Taylor type expansion (3.7) can be rewritten as

$$
\begin{aligned}
& f^{T}(x, y, z, s) \\
= & a_{111}(s) \frac{\left(x-x_{0}\right)^{3}}{3 !}+a_{222}(s) \frac{\left(y-y_{0}\right)^{3}}{3 !}+a_{333}(s) \frac{\left(z-z_{0}\right)^{3}}{3 !} \\
& +3 a_{112}(s) \frac{\left(x-x_{0}\right)^{2}}{2 !}\left(y-y_{0}\right)+3 a_{122}(s)\left(x-x_{0}\right) \frac{\left(y-y_{0}\right)^{2}}{2 !} \\
& +3 a_{113}(s) \frac{\left(x-x_{0}\right)^{2}}{2 !}\left(z-z_{0}\right)+3 a_{223}(s) \frac{\left(y-y_{0}\right)^{2}}{2 !}\left(z-z_{0}\right) \\
& +3 a_{133}(s)\left(x-x_{0}\right) \frac{\left(z-z_{0}\right)^{2}}{2 !}+3 a_{233}(s)\left(y-y_{0}\right) \frac{\left(z-z_{0}\right)^{2}}{2 !} \\
& +6 a_{123}(s)\left(x-x_{0}\right)\left(y-y_{0}\right)\left(z-z_{0}\right) \\
& +a_{11}(s) \frac{\left(x-x_{0}\right)^{2}}{2 !}+a_{22}(s) \frac{\left(y-y_{0}\right)^{2}}{2 !}+a_{33}(s) \frac{\left(z-z_{0}\right)^{2}}{2 !} \\
& +2 a_{12}(s)\left(x-x_{0}\right)\left(y-y_{0}\right)+2 a_{13}(s)\left(x-x_{0}\right)\left(z-z_{0}\right)+2 a_{23}(s)\left(y-y_{0}\right)\left(z-z_{0}\right) \\
& +a_{1}(s)\left(x-x_{0}\right)+a_{2}(s)\left(y-y_{0}\right)+a_{3}(s)\left(z-z_{0}\right)+f^{T}\left(x_{0}, y_{0}, z_{0}, s\right) .
\end{aligned}
$$

Using the triply cubic expansion (3.9), the component $p_{i j k}$ of the vector function $\mathbf{p}_{i j k}\left(x_{1}, x_{2}, x_{3}, s\right)$ can be expressed at per cubic lattice $C_{i j k}$ in the form of:

$$
\begin{aligned}
& \mathbf{p}_{i j k}(x, y, z, s) \approx p_{i j k}^{n}(x, y, z, s) \\
= & a_{111}(s) \frac{\left(x-x_{i-1}\right)^{3}}{3 !}+a_{222}(s) \frac{\left(y-y_{j-1}\right)^{3}}{3 !}+a_{333}(s) \frac{\left(z-z_{k-1}\right)^{3}}{3 !} \\
& +3 a_{112}(s) \frac{\left(x-x_{i-1}\right)^{2}}{2 !}\left(y-y_{j-1}\right)+3 a_{122}(s)\left(x-x_{i-1}\right) \frac{\left(y-y_{j-1}\right)^{2}}{2 !} \\
& +3 a_{113}(s) \frac{\left(x-x_{i-1}\right)^{2}}{2 !}\left(z-z_{k-1}\right)+3 a_{223}(s) \frac{\left(y-y_{j-1}\right)^{2}}{2 !}\left(z-z_{k-1}\right) \\
& +3 a_{133}(s)\left(x-x_{i-1}\right) \frac{\left(z-z_{k-1}\right)^{2}}{2 !}+3 a_{233}(s)\left(y-y_{j-1}\right) \frac{\left(z-z_{k-1}\right)^{2}}{2 !} \\
& +6 a_{123}(s)\left(x-x_{i-1}\right)\left(y-y_{j-1}\right)\left(z-z_{k-1}\right) \\
& +a_{11}(s) \frac{\left(x-x_{i-1}\right)^{2}}{2 !}+a_{22}(s) \frac{\left(y-y_{j-1}\right)^{2}}{2 !}+a_{33}(s) \frac{\left(z-z_{k-1}\right)^{2}}{2 !} \\
& +2 a_{12}(s)\left(x-x_{i-1}\right)\left(y-y_{j-1}\right)+2 a_{13}(s)\left(x-x_{i-1}\right)\left(z-z_{k-1}\right) \\
& +2 a_{23}(s)\left(y-y_{j-1}\right)\left(z-z_{k-1}\right)+a_{1}(s)\left(x-x_{i-1}\right)+a_{2}(s)\left(y-y_{j-1}\right) \\
& +a_{3}(s)\left(z-z_{k-1}\right)+p_{i j k}^{n}\left(x_{i-1}, y_{j-1}, z_{k-1}, s\right)
\end{aligned}
$$

for $i=1,2, \cdots, N, j=1,2, \cdots, N, k=1,2, \cdots, N$ and $n=1,2, \cdots, \mathbf{n}$. Via the Taylor type expansion (3.10) of $\mathbf{p}_{i j k}^{n}$ at point $\left(x_{0}, y_{0}, z_{0}, s\right)$ with the coefficients as in (3.8), it is naturally to assume the $a_{i j k}(s), a_{i j}(s), a_{i}(s)$, which need be identified, take the form of

$$
\begin{aligned}
& a_{i}(s)=\alpha_{i} s+\alpha_{0}, \quad i=1,2,3 \\
& a_{i j}(s)=\beta_{i j} s^{2}+\beta_{i} s+\beta_{0}, \quad i=1,2,3 . j=1,2,3 . \\
& a_{i j k}(s)=\gamma_{i j k} s^{3}+\gamma_{i j} s^{2}+\gamma_{i} s+\gamma_{0}, \quad i=1,2,3 . j=1,2,3 . k=1,2,3 .
\end{aligned}
$$


for constants $\alpha_{i}, \alpha_{0} \in R^{1}, \beta_{i j}, \beta_{i}, \beta_{0} \in R^{1}$ and $\gamma_{i j k}, \gamma_{i j}, \gamma_{i}, \gamma_{0} \in R^{1}$, where $R^{1}$ is real number set. The indeterminated coefficient constants in (3.11), (3.12) and (3.13) can be calculated at each cubic lattice $C_{i j k}$.

Remark 3.1. Clearly, the idea can be extended to different polynomials expansion.

Remark 3.2. The comparison with existing methods is not considered into this paper yet. To compare the convergence order, error estimation and occupied CPU memory usage with indirect approach are left to future works.

\subsection{Objective function minimization}

Citing [3] to do the minimization for a proper objective function given in the form of quadratic criteria as

$$
J(\mathbf{p})=\int_{0}^{\infty} \int_{\Omega}|\mathbf{L}(\mathbf{p}(\mathbf{x}, t))|^{2} C_{\lambda}^{2}(\mathbf{x}) d \mathbf{x} d t .
$$

That is

$$
J(\mathbf{p})=\int_{0}^{\infty} \int_{0}^{L} \int_{0}^{L} \int_{0}^{L}|\mathbf{L}(\mathbf{p}(x, y, z, t))|^{2} C_{\lambda}^{2}(x, y, z) d x d y d z d t
$$

where Carleman's weighted function $C_{\lambda}(x, y, z)=\exp (-\lambda x-\lambda y-\lambda z)$, which appeared in Carleman estimates (cf. [17]) for the operators $\frac{d^{2}}{d x^{2}}, \frac{d^{2}}{d y^{2}}, \frac{d^{2}}{d z^{2}}$, and $\lambda$ is parameter. Corresponding to partition $C_{i j k}$ on spatial domain $\Omega$, the criteria functional (3.14) is equivalent to

$$
J(\mathbf{p})=\lim _{\bar{s} \rightarrow \infty} \sum_{i=1}^{N} \sum_{j=1}^{N} \sum_{k=1}^{N} J_{\lambda, i, j, k}(\mathbf{p}),
$$

where resulting functional in (3.15) at each cubic lattice with respect to the $(i, j, k)$-th coefficients $\alpha_{i}(s), \alpha_{i j}(s), \alpha_{i j k}(s)$ of the triply cubic polynomials is:

$$
J_{\lambda, i, j, k}(\mathbf{p})=\int_{s_{0}}^{\bar{s}} \int_{z_{k-1}}^{z_{k}} \int_{y_{j-1}}^{y_{j}} \int_{x_{i-1}}^{x_{i}}\left|\mathbf{L}\left(\mathbf{p}_{i j k}(x, y, z, t)\right)\right|^{2} C_{\lambda, i, j, k}^{2}(x, y, z) d x d y d z d t
$$

where $C_{\lambda, i, j, k}(x, y, z)=\exp \left[-\lambda\left(x-x_{i-1}\right)-\lambda\left(y-y_{j-1}\right)-\lambda\left(z-z_{k-1}\right)\right]$ are Carleman's weighted function, and $s_{0}=0$ or $s_{0}>0$. The calculation of $J_{\lambda, i, j, k}$ can be implemented by using Gauss-Legendre quadrature (cf. [3, 18]) as

$$
\hat{J}_{\lambda, \mathbf{i}, \mathbf{j}, \mathbf{k}}=\sum_{\mathbf{t}=1}^{\mathbf{T}} w_{\mathbf{t}} \sum_{\mathbf{i}=1}^{\mathbf{I}} w_{\mathbf{i}} \sum_{\mathbf{j}=1}^{\mathbf{J}} w_{\mathbf{j}} \sum_{\mathbf{k}=1}^{\mathbf{K}} w_{\mathbf{k}}\left|\mathbf{L}\left(\mathbf{p}_{i j k}\left(x_{\mathbf{i}}, y_{\mathbf{j}}, z_{\mathbf{k}}, \mathbf{t}\right)\right)\right|^{2} C_{\lambda, \mathbf{i}, \mathbf{j}, \mathbf{k}}^{2}\left(x_{\mathbf{i}}, y_{\mathbf{j}}, z_{\mathbf{k}}\right),
$$

where $w_{\mathbf{i}}, w_{\mathbf{j}}, w_{\mathbf{k}}, w_{\mathbf{t}}$ are Gauss weights, and $x_{\mathbf{i}}, y_{\mathbf{j}}, z_{\mathbf{k}}, \mathbf{t}$ are Gauss points at $x, y, z$ and $t$ direction, respectively. $\mathbf{T}, \mathbf{I}, \mathbf{J}, \mathbf{K}$ are the maximum number of indices $\mathbf{t}, \mathbf{i}, \mathbf{j}, \mathbf{k}$ for Gauss points at each direction $t, x, y, z$. Take the usual norms as

$$
\left\|a_{i}\right\|=\max _{s \in\left[s_{0}, \bar{s}\right]}\left|a_{i}(s)\right|,\left\|a_{i j}\right\|=\max _{s \in\left[s_{0}, \bar{s}\right]}\left|a_{i j}(s)\right|,\left\|a_{i j k}\right\|=\max _{s \in\left[s_{0}, \bar{s}\right]}\left|a_{i j k}(s)\right| .
$$


Use the notation $\mathrm{n}$ without confusion with $n$. The sequential minimizer of $J_{\lambda, i, j, k}\left(p_{i j k}^{n}\right)$ can be searched on a bounded set

$$
G_{i j k}(\mathrm{n})=\left\{\left(a_{i}(s), a_{i j}(s), a_{i j k}(s)\right) \mid\left\|a_{i}\right\| \leq \mathrm{n},\left\|a_{i j}\right\| \leq \mathrm{n},\left\|a_{i j k}\right\| \leq \mathrm{n}\right\}
$$

for a positive number $\mathrm{n}>0$. The corresponding set of $p_{i j k}^{n}$ is denoted by

$$
G(\mathrm{n})=\left\{p_{i j k}^{n}(s) \mid\left\|p_{i j k}^{n}\right\| \leq \mathrm{n}\right\} .
$$

Using the result of [11] and the convexity of $J_{\lambda, i, j, k}(\mathbf{p})$, the uniqueness of minimizer for the functional (3.16) and the convergence of posed approach can be proven.

Definition 3.3. Suppose that there exists a vector function $\mathbf{p}^{*}(x, y, z, s)$, such that

$$
\left\{\begin{array}{l}
\hat{\mathbf{L}}\left(\mathbf{p}^{*}\right)=0, \mathbf{p}^{*}=\mathbf{p}^{*}(x, y, z, s), x \in(0, L), y \in(0, L), z \in(0, L), s \in\left(s_{0}, \bar{s}\right), \\
\frac{\partial \mathbf{p}^{*}(x, y, z, s)}{\partial \eta}+a \mathbf{p}^{*}(x, y, z, s)=\mathbf{p}_{0}^{*}(s), \quad(x, y, z) \in \Gamma .
\end{array}\right.
$$

where

$$
\hat{\mathbf{L}}\left(\mathbf{p}^{*}\right) \equiv F^{*}\left(\int_{s}^{\bar{s}} \mathbf{p}^{* \prime \prime}(x, y, z, \nu) d \nu, \int_{s}^{\bar{s}} \mathbf{p}^{* \prime}(x, y, z, \nu) d \nu, x, y, z, s\right) .
$$

Then $\mathbf{p}^{*}(x, y, z, s)$ is called the exact solution to (3.5).

Assume that $\mathbf{p}^{*} \in C^{3}(\Omega)$ and satisfy

$$
\max _{s \in\left[s_{0}, \bar{s}\right]}\left\|\mathbf{p}^{*}(x, y, z, s)\right\|_{C^{3}(\Omega)} \leq c_{0} \mathrm{n},
$$

where $c_{0}$ is constant. It means that $\mathbf{p}^{*} \in G\left(c_{0} \mathbf{n}\right)$. The function $F$ is approximately known as

$$
\begin{aligned}
& F\left(\int_{s}^{\bar{s}} \mathbf{p}^{\prime \prime}(x, y, z, \nu) d \nu, \int_{s}^{\bar{s}} \mathbf{p}^{\prime}(x, y, z, \nu) d \nu, x, y, z, s\right) \\
= & \left.F^{*}\left(\int_{s}^{\bar{s}} \mathbf{p}^{* \prime \prime}(x, y, z, \nu) d \nu, \int_{s}^{\bar{s}} \mathbf{p}^{* \prime}(x, y, z, \nu) d \nu, x, y, z, s\right)\right)+\hat{\varepsilon}(x, y, z, s),
\end{aligned}
$$

where $\hat{\varepsilon}(x, y, z, s)$ is error function, and

$$
\max _{s \in\left[s_{0}, \bar{s}\right]}\|\hat{\varepsilon}(x, y, z, s)\|_{C^{3}(\Omega)} \leq \varepsilon, \max _{s \in\left[s_{0}, \bar{s}\right]}\left|\mathbf{p}_{0}(s)-\mathbf{p}_{0}^{*}(s)\right| \leq \varepsilon
$$

for a constant $\varepsilon$. At each node $\left(x_{i-1}, y_{j-1}, z_{k-1}\right)$, denote that $F$ and $F^{*}$ take the form of

$$
\begin{aligned}
& F_{i j k}=\left.F\left(\int_{s}^{\bar{s}} \mathbf{p}_{i j k}^{\prime \prime}(x, y, z, \nu) d \nu, \int_{s}^{\bar{s}} \mathbf{p}_{i j k}^{\prime}(x, y, z, \nu) d \nu, x, y, z, s\right)\right|_{\begin{array}{l}
x=x_{i-1}, \\
y=y_{j-1}, \\
z=z_{k-1}
\end{array}} \\
& F_{i j k}^{*}=F\left(\int_{s}^{\bar{s}} \mathbf{p}_{i j k}^{* \prime \prime}(x, y, z, \nu) d \nu, \int_{s}^{\bar{s}} \mathbf{p}_{i j k}^{* \prime}(x, y, z, \nu) d \nu, x, y, z, s\right) \mid \begin{array}{l}
x=x_{i-1} \\
y=y_{j-1}, \\
z=z_{k-1}
\end{array}
\end{aligned}
$$


Theorem 3.4. Assume that above assumptions are satisfied. Let $\left(\alpha_{0}, \alpha_{00}, \alpha_{000}\right)$, $\left(\alpha_{i}, \alpha_{i j}, \alpha_{i j k}\right)$ and $\left(\alpha_{i}^{\prime}, \alpha_{i j}^{\prime}, \alpha_{i j k}^{\prime}\right)$ be the corresponding coefficients of $p_{0}^{n}, p_{i j k}^{n}$ and $p_{i j k}^{n \prime}$, respectively. Let $h_{i}, h_{j}, h_{k}$ and $h_{i 0}, h_{j 0}, h_{k 0}$ be the discrete lengths of the subintervals at $x, y$ and $z$ direction, respectively. Assume that $\left(\alpha_{0}, \alpha_{00}, \alpha_{000}\right) \in G_{i j k}(\mathrm{n})$. Then there exist 3 sufficiently-small positive numbers $h_{i 0}(\mathrm{n}), h_{j 0}(\mathrm{n})$ and $h_{k 0}(\mathrm{n})$ such that for all $h_{i} \in\left(0, h_{i 0}\right), h_{j} \in\left(0, h_{j 0}\right)$ and $h_{k} \in\left(0, h_{k 0}\right)$

$$
\begin{aligned}
& \left(\alpha_{i}, \alpha_{i j}, \alpha_{i j k}\right),\left(\alpha_{i}^{\prime}, \alpha_{i j}^{\prime}, \alpha_{i j k}^{\prime}\right) \in G_{i j k}(\mathrm{n}), \\
& p_{i j k}^{n}\left(x_{i-1}, y_{j-1}, z_{k-1}, s\right), p_{i j k}^{\prime \prime}\left(x_{i-1}, y_{j-1}, z_{k-1}, s\right) \in G(\mathrm{n}), \\
& F_{i j k} \in G\left(c_{0} \mathrm{n}\right), \quad i=1,2, \ldots, N, \quad j=1,2, \ldots, N, k=1,2, \ldots, N .
\end{aligned}
$$

All the functionals $J_{\lambda, i, j, k}(\mathbf{p})$ are strictly convex on $G(\mathrm{n})$. The unique minimizer pair $\left(\alpha_{i}^{*}, \alpha_{i j}^{*}, \alpha_{i j k}^{*}\right)$ of $J_{\lambda, i, j, k}\left(\mathbf{p}^{*}\right)$ is an interior point of $G_{i j k}(\mathbf{n})$. It can be regarded as the solution of operation equation $\Psi_{\lambda, i, j, k}\left(\alpha_{i}, \alpha_{i j}, \alpha_{i j k}\right)=\left(\alpha_{i}^{*}, \alpha_{i j}^{*}, \alpha_{i j k}^{*}\right)$ with the map: $\Psi_{\lambda, i, j, k}: G_{i j k}(\mathrm{n}) \rightarrow G_{i j k}(\mathrm{n})$. Its corresponding solution $p_{i j k}^{* n}(x, y, z, s)$ is satisfying operator equation $\Phi_{\lambda, i, j, k} p_{i j k}^{n}(x, y, z, s)=p_{i j k}^{* n}(x, y, z, s)$ with map: $\Phi_{\lambda, i, j, k}: G(\mathrm{n}) \rightarrow G(\mathrm{n})$. Further, operator equation $\Xi_{i j k} F_{i j k}=F_{i j k}^{*}$ is satisfied for map $\Xi_{i j k}: G\left(c_{0} \mathrm{n} \rightarrow G\left(c_{0} \mathrm{n}\right)\right.$.

Citing [1], [2] and [11] to get the complete proof. It is omitted in here.

Theorem 3.4 evident that the convergence of the triply cubic polynomials approach to the exact solution is guaranteed for initial guess $\left(\alpha_{0}, \alpha_{00}, \alpha_{000}\right)$ in the set $G_{i j k}(\mathrm{n})$. Further, it briefly verified that the globally convergence of proposed triply cubic polynomials approach in three dimension. To render the details in Section 4.

Theorem 3.5. If the equivalently approximate field $\tilde{f}(\mathbf{x}, \nu)$ can be founded, the unknown coefficient $k(\mathbf{x})$ is calculated by the expression (2.3). The inversion formula for $\tilde{k}(\mathbf{x})$ is deduced as

$$
\tilde{k}(\mathbf{x})=\nabla^{2} \tilde{f}+(\nabla \tilde{f})^{2}-s, \quad \forall s \in\left[s_{0}, \bar{s}\right] .
$$

Obviously, there is no need to compute the solutions for getting the $\tilde{k}(\mathbf{x})$ (cf. [11]).

\section{Convergence and error estimate}

\subsection{Convergence order}

Theorem 4.1. If all assumptions are held, then the triply cubic polynomials approach is globally converged with the order of $o\left(h^{3}\right)$.

Proof. Firstly, in one coordinate $\chi$ case, let $f_{\tau}(\chi)$ (the index ' $\tau$ ' denote the 'Taylor expansion') be a function on the interval $\chi \in[a, b]$. The remainder of the Taylor type expansion is as follow:

$$
R_{\tau}=\int_{a}^{b} f_{\tau}(\chi) d \chi-\sum_{n=1}^{\mathbf{N}} A_{n} f_{\tau}\left(\chi_{n}\right)=\frac{f_{\tau}^{(\mathbf{N}+1)}(\bar{\chi})}{(\mathbf{N}+1) !} \int_{a}^{b} w^{2}(\chi) d \chi
$$


where $w(\chi)=\left(\chi-\chi_{1}\right)\left(\chi-\chi_{2}\right) \cdots\left(\chi-\chi_{\mathbf{n}}\right), a<\bar{\chi}<b$. The interval $[a, b]$ is taken $[s, \bar{s}]$ as integral intervals and $\chi$ is $d \nu$ as variable. In our case $\mathbf{N}=3$ for cubic polynomials $\mathbf{p}_{i j k}$, the convergence order is $o\left(h^{3}\right)$ if setting $h_{i}=h_{j}=h_{k}=h$ for triple integrals.

Secondly, for a function $g(\chi)$, the error estimate of Gauss-Legendre (GL) quadrature for the interval $[-1,1]$ is as follows:

$$
E_{G}=\int_{-1}^{1} g(\chi) d \chi-\sum_{k=1}^{\kappa} \frac{2 g\left(\xi_{k}^{(\kappa)}\right)}{\left(1-\xi_{k}^{(\kappa)}\right)\left[p_{\kappa}^{\prime}\left(\xi_{k}^{(\kappa)}\right]^{2}\right.}+\frac{2^{2 \kappa+1}(\kappa !)^{4}}{(2 \kappa+1)[(2 \kappa) !]^{3}} g^{(2 \kappa)}(\bar{\chi}),
$$

where $\xi_{k}^{(\kappa)}$ are the roots of the GL polynomials $p_{\kappa}(\chi)$, and $-1<\bar{\chi}<1$. For regularity, it need to transform the interval $[-1,1]$ to $\left[x_{i-1}, x_{i}\right],\left[y_{j-1}, y_{j}\right],\left[z_{k-1}, z_{k}\right]$ and $\left[s_{0}, \bar{s}\right]$ at $(3.17)$. Clearly, the convergence order is $o\left(h^{2 \kappa}\right)$. In the case $\kappa=6$ (usual take Gauss points $\mathbf{I}=\mathbf{J}=\mathbf{K}=\mathbf{T}=6$ in numerical experiments) for four variables $x, y, z, \nu$ at (3.17), the convergence order is $o\left(h^{12}\right)$ of $\hat{J}$ if setting $h_{i}=h_{j}=h_{k}=h$ as triple integral intervals and $h_{t}=h$ as $t$ integral interval.

Combining the results of (4.1) and (4.2), roughly, we obtain the convergence order of our proposed globally convergent algorithm at each cubic lattice as

$$
\mathbf{p}(x, y, z, s) \approx \mathbf{p}_{i j k}(x, y, z, s)+o\left(h^{3}\right), \quad J_{\lambda, i, j, k}(\mathbf{p}) \approx \hat{J}_{\lambda, i, j, k}(\mathbf{p})+o\left(h^{12}\right) .
$$

Therefore, the total convergence order is deduced by

$$
\begin{aligned}
& \left|J_{\lambda, i, j, k}(\mathbf{p})-\hat{J}_{\lambda, i, j, k}\left(\mathbf{p}_{i j k}\right)\right| \\
\leq & \mid \int_{s_{0}}^{\bar{s}} \int_{z_{j-1}}^{z_{j}} \int_{y_{j-1}}^{y_{j}}\left[\int_{x_{i-1}}^{x_{i}}|\mathbf{L}(\mathbf{p}(x, y, z, t))|^{2} C_{\lambda, i, j, k}^{2}(x, y, z) d x\right. \\
& \left.-\sum_{\mathbf{i}=1}^{\mathbf{I}} w_{\mathbf{i}}\left|\mathbf{L}\left(\mathbf{p}\left(x_{\mathbf{i}}, y, z, t\right)\right)\right|^{2} C_{\lambda, i, j}^{2}\left(x_{\mathbf{i}}, y, z\right)\right] d y d z d t \mid \\
& +\mid \int_{s_{0}}^{\bar{s}} \int_{z_{j-1}}^{z_{j}}\left[\int_{y_{j-1}}^{y_{j}} \sum_{i i=1}^{\mathbf{I}} w_{\mathbf{i}}\left|\mathbf{L}\left(\mathbf{p}\left(x_{\mathbf{i}}, y, z, t\right)\right)\right|^{2} C_{\lambda, i, j}^{2}\left(x_{\mathbf{i}}, y, z\right) d y\right. \\
& \left.-\sum_{\mathbf{j}=1}^{\mathbf{J}} w_{\mathbf{j}} \sum_{\mathbf{i}=1}^{\mathbf{I}} w_{\mathbf{i}}\left|\mathbf{L}\left(\mathbf{p}\left(x_{\mathbf{i}}, y_{\mathbf{j}}, z, t\right)\right)\right|^{2} C_{\lambda, i, j}^{2}\left(x_{\mathbf{i}}, y_{\mathbf{j}}, z\right)\right] d z d t \mid \\
& +\mid \int_{s_{0}}^{\bar{s}}\left[\int_{z_{j-1}}^{z_{j}} \sum_{\mathbf{j}=1}^{\mathbf{J}} w_{\mathbf{j}} \sum_{\mathbf{i}=1}^{\mathbf{I}} w_{\mathbf{i}}\left|\mathbf{L}\left(\mathbf{p}\left(x_{\mathbf{i}}, y_{\mathbf{j}}, z, t\right)\right)\right|^{2} C_{\lambda, i, j}^{2}\left(x_{\mathbf{i}}, y_{\mathbf{j}}, z\right) d z\right. \\
& \left.-\sum_{\mathbf{k}=1}^{\mathbf{K}} w_{\mathbf{k}} \sum_{\mathbf{j}=1}^{\mathbf{J}} w_{\mathbf{j}} \sum_{\mathbf{i}=1}^{\mathbf{I}} w_{\mathbf{i}}\left|\mathbf{L}\left(\mathbf{p}\left(x_{\mathbf{i}}, y_{\mathbf{j}}, z_{\mathbf{k}}, t\right)\right)\right|^{2} C_{\lambda, i, j}^{2}\left(x_{\mathbf{i}}, y_{\mathbf{j}}, z_{\mathbf{k}}\right)\right] d t \mid \\
+ & \left.\left|\int_{s_{0}}^{\bar{s}} \sum_{\mathbf{k}=1}^{\mathbf{K}} w_{\mathbf{k}} \sum_{\mathbf{j}=1}^{\mathbf{J}} w_{\mathbf{j}} \sum_{\mathbf{i}=1}^{\mathbf{I}} w_{\mathbf{i}}\right| \mathbf{L}\left(\mathbf{p}\left(x_{\mathbf{i}}, y_{\mathbf{j}}, z_{\mathbf{k}}, t\right)\right)\right|^{2} C_{\lambda, i, j}^{2}\left(x_{\mathbf{i}}, y_{\mathbf{j}}, z_{\mathbf{k}}\right) d t
\end{aligned}
$$




$$
\begin{aligned}
& -\sum_{\mathbf{t}=1}^{\mathbf{T}} w_{\mathbf{t}} \sum_{\mathbf{k}=1}^{\mathbf{K}} w_{\mathbf{k}} \sum_{\mathbf{j}=1}^{\mathbf{J}} w_{\mathbf{j}} \sum_{\mathbf{i}=1}^{\mathbf{I}} w_{\mathbf{i}}\left|\mathbf{L}\left(\mathbf{p}\left(x_{\mathbf{i}}, y_{\mathbf{j}}, z_{\mathbf{k}}, \mathbf{t}\right)\right)\right|^{2} C_{\lambda, i, j, k}^{2}\left(x_{\mathbf{i}}, y_{\mathbf{j}}, z_{\mathbf{k}}\right) \mid \\
& +\left|\hat{J}_{\lambda, i, j, k}(\mathbf{p})-\hat{J}_{\lambda, i, j, k}\left(\mathbf{p}_{i j k}\right)\right| \\
\approx & O\left(h^{2 \mathbf{I}+1}\right)+O\left(h^{2 \mathbf{J}+1}\right)+O\left(h^{2 \mathbf{K}+1}\right)+O\left(h^{2 \mathbf{T}+1}\right)+o\left(h^{12}\right) \approx o\left(h^{12}\right) .
\end{aligned}
$$

Consequently, the triply cubic polynomials approach induced globally convergence order is $o\left(h^{12}\right)$. It completes the proof of Theorem 4.1 .

\subsection{Error estimate}

Consider the error estimate of proposed triply cubic approach. The exact solution function $\mathbf{p}^{*}(x, y, z, s)$ in Definition 3.3 can be expanded using Taylor type series at each cube $C_{i j k}$ with the exact form and remainder as

$$
\begin{aligned}
& \mathbf{p}^{*}(x, y, z, s)=a_{111}^{*}(s) \frac{\left(x-x_{i-1}\right)^{3}}{3 !}+a_{222}^{*}(s) \frac{\left(y-y_{j-1}\right)^{3}}{3 !}+a_{333}^{*}(s) \frac{\left(z-z_{k-1}\right)^{3}}{3 !} \\
& +3 a_{112}^{*}(s) \frac{\left(x-x_{i-1}\right)^{2}}{2 !}\left(y-y_{j-1}\right)+3 a_{122}^{*}(s)\left(x-x_{i-1}\right) \frac{\left(y-y_{j-1}\right)^{2}}{2 !} \\
& +3 a_{113}^{*}(s) \frac{\left(x-x_{i-1}\right)^{2}}{2 !}\left(z-z_{k-1}\right)+3 a_{223}^{*}(s) \frac{\left(y-y_{j-1}\right)^{2}}{2 !}\left(z-z_{k-1}\right) \\
& +3 a_{133}^{*}(s)\left(x-x_{i-1}\right) \frac{\left(z-z_{k-1}\right)^{2}}{2 !}+3 a_{233}^{*}(s)\left(y-y_{j-1}\right) \frac{\left(z-z_{k-1}\right)^{2}}{2 !} \\
& +6 a_{123}^{*}(s)\left(x-x_{i-1}\right)\left(y-y_{j-1}\right)\left(z-z_{k-1}\right) \\
& +a_{11}^{*}(s) \frac{\left(x-x_{i-1}\right)^{2}}{2 !}+a_{22}^{*}(s) \frac{\left(y-y_{j-1}\right)^{2}}{2 !}+a_{33}^{*}(s) \frac{\left(z-z_{k-1}\right)^{2}}{2 !} \\
& +2 a_{12}^{*}(s)\left(x-x_{i-1}\right)\left(y-y_{j-1}\right)+2 a_{13}^{*}(s)\left(x-x_{i-1}\right)\left(z-z_{k-1}\right) \\
& +2 a_{23}^{*}(s)\left(y-y_{j-1}\right)\left(z-z_{k-1}\right) \\
& +a_{1}^{*}(s)\left(x-x_{i-1}\right)+a_{2}^{*}(s)\left(y-y_{j-1}\right)+a_{3}^{*}(s)\left(z-z_{k-1}\right) \\
& +p_{i j k}\left(x_{i-1}, y_{j-1}, z_{k-1}, s\right)+R_{s} \frac{\left(\bar{x}-x_{i-1}\right)^{4}+\left(\bar{y}-y_{j-1}\right)^{4}+\left(\bar{z}-z_{k-1}\right)^{4}}{4 !} .
\end{aligned}
$$

In (4.4), $R_{s}=p^{*(4)}(\bar{x}, \bar{y}, \bar{z}, s)$ is remainder of the Taylor type expansion for exact solution $\mathbf{p}^{*}(x, y, z, s)$, and $x_{i-1}<\bar{x}<x_{i}, y_{j-1}<\bar{y}<y_{j}, z_{k-1}<\bar{z}<z_{k}$. By (4.1), (4.2) and (4.3) to get the error estimate as

$$
\begin{aligned}
& \left|J_{\lambda, i, j, k}\left(\mathbf{p}^{*}\right)-\hat{J}_{\lambda, i, j, k}\left(\mathbf{p}_{i j k}\right)\right| \\
\leq & \left|J_{\lambda, i, j, k}(\mathbf{p})-\hat{J}_{\lambda, i, j, k}\left(\mathbf{p}^{*}\right)\right|+\left|\hat{J}_{\lambda, i, j, k}\left(\mathbf{p}^{*}\right)-\hat{J}_{\lambda, i, j, k}\left(\mathbf{p}_{i j k}\right)\right| \\
\leq & \mid \sum_{\mathbf{t}=1}^{\mathbf{T}} w_{\mathbf{t}} \sum_{\mathbf{k}=1}^{\mathbf{K}} w_{\mathbf{k}} \sum_{\mathbf{j}=1}^{\mathbf{J}} w_{\mathbf{j}} \sum_{\mathbf{i}=1}^{\mathbf{I}} w_{\mathbf{i}}\left(\left|\mathbf{L}\left(\mathbf{p}^{*}\left(x_{\mathbf{i}}, y_{\mathbf{j}}, z_{\mathbf{k}}, \mathbf{t}\right)\right)\right|^{2}-\left|\mathbf{L}\left(\mathbf{p}_{i j k}\left(x_{\mathbf{i}}, y_{\mathbf{j}}, z_{\mathbf{k}}, \mathbf{t}\right)\right)\right|^{2}\right) \\
& C_{\lambda, i, j, k}^{2}\left(x_{\mathbf{i}}, y_{\mathbf{j}}, z_{\mathbf{k}}\right) \mid+\frac{2^{2 \kappa+1}(\kappa !)^{4}}{(2 \kappa+1)[(2 \kappa) !]^{3}} f^{(2 \kappa)}\left(\theta_{x}, \theta_{y}, \theta_{z}, \tau\right),
\end{aligned}
$$


where $f\left(\theta_{x}, \theta_{y}, \theta_{z}, \tau\right)=\left|\mathbf{L}\left(\mathbf{p}_{i j k}\left(\theta_{x}, \theta_{y}, \theta_{z}, \tau\right)\right)\right|^{2} C_{\lambda, i, j, k}^{2}\left(\theta_{x}, \theta_{y}, \theta_{z}\right)$ and $x_{\mathbf{i}-1}<\theta_{x}<x_{\mathbf{i}}$, $y_{\mathbf{j}-1}<\theta_{y}<y_{\mathbf{j}}, z_{\mathbf{k}-1}, \theta_{z}<z_{\mathbf{k}}$ and $\mathbf{t}-1<\tau<\mathbf{t}$. For the functional $\mathbf{L}(\mathbf{p})$ of (4.5), from Taylor type expansion (3.7), (3.18), (3.19) and exact expansion (4.4), one can estimate that

$$
\begin{aligned}
& \left|\mathbf{L}\left(\mathbf{p}^{*}\left(x_{\mathbf{i}}, y_{\mathbf{j}}, z_{\mathbf{k}}, t\right)\right)\right|^{2}-\left|\mathbf{L}\left(\mathbf{p}_{i j k}\left(x_{\mathbf{i}}, y_{\mathbf{j}}, z_{\mathbf{k}}, t\right)\right)\right|^{2} \\
= & \left|F\left(\int_{s}^{\bar{s}} \mathbf{p}^{* \prime \prime}\left(x_{\mathbf{i}}, y_{\mathbf{j}}, z_{\mathbf{k}}, \nu\right) d \nu, \int_{s}^{\bar{s}} \mathbf{p}^{* \prime}\left(x_{\mathbf{i}}, y_{\mathbf{j}}, z_{\mathbf{k}}, \nu\right) d \nu, x_{\mathbf{i}}, y_{\mathbf{j}}, z_{\mathbf{k}}, s\right)\right|^{2} \\
& -\left|F\left(\int_{s}^{\bar{s}} \mathbf{p}_{i j k}^{\prime \prime}\left(x_{\mathbf{i}}, y_{\mathbf{j}}, z_{\mathbf{k}}, \nu\right) d \nu, \int_{s}^{\bar{s}} \mathbf{p}_{i j k}^{\prime}\left(x_{\mathbf{i}}, y_{\mathbf{j}}, z_{\mathbf{k}}, \nu\right) d \nu, x_{\mathbf{i}}, y_{\mathbf{j}}, z_{\mathbf{k}}, s\right)\right|^{2} \\
= & \mid F^{*}\left(\int_{s}^{\bar{s}} \mathbf{p}^{* \prime \prime}\left(x_{\mathbf{i}}, y_{\mathbf{j}}, z_{\mathbf{k}}, \nu\right) d \nu, \int_{s}^{\bar{s}} \mathbf{p}^{* \prime}\left(x_{\mathbf{i}}, y_{\mathbf{j}}, z_{\mathbf{k}}, \nu\right) d \nu, x_{\mathbf{i}}, y_{\mathbf{j}}, z_{\mathbf{k}}, s\right) \\
& +F_{i j k}\left(\int_{s}^{\bar{s}} \mathbf{p}_{i j k}^{\prime \prime}\left(x_{\mathbf{i}}, y_{\mathbf{j}}, z_{\mathbf{k}}, \nu\right) d \nu, \int_{s}^{\bar{s}} \mathbf{p}_{i j k}^{\prime}\left(x_{\mathbf{i}}, y_{\mathbf{j}}, z_{\mathbf{k}}, \nu\right) d \nu, x_{\mathbf{i}}, y_{\mathbf{j}}, z_{\mathbf{k}}, s\right) \mid \\
& \times \mid F^{*}\left(\int_{s}^{\bar{s}} \mathbf{p}^{* \prime \prime}\left(x_{\mathbf{i}}, y_{\mathbf{j}}, z_{\mathbf{k}}, \nu\right) d \nu, \int_{s}^{\bar{s}} \mathbf{p}^{* \prime}\left(x_{\mathbf{i}}, y_{\mathbf{j}}, z_{\mathbf{k}}, \nu\right) d \nu, x_{\mathbf{i}}, y_{\mathbf{j}}, z_{\mathbf{k}}, s\right) \\
& -F_{i j k}\left(\int_{s}^{\bar{s}} \mathbf{p}_{i j k}^{\prime}\left(x_{\mathbf{i}}, y_{\mathbf{j}}, z_{\mathbf{k}}, \nu\right) d \nu, \int_{s}^{s} \mathbf{p}_{i j k}^{\prime}\left(x_{\mathbf{i}}, y_{\mathbf{j}}, z_{\mathbf{k}}, \nu\right) d \nu, x_{\mathbf{i}}, y_{\mathbf{j}}, z_{\mathbf{k}}, s\right) \mid \\
\leq & 4 c_{0} n^{\prime \prime} \hat{\varepsilon} .
\end{aligned}
$$

Error $\hat{\varepsilon}\left(x_{\mathbf{i}}, y_{\mathbf{j}}, z_{\mathbf{k}}, \mathbf{t}\right)$ in (4.6) is a sufficiently-small number depended on the node $\left(x_{\mathbf{i}}, y_{\mathbf{j}}, z_{\mathbf{k}}\right)$ of $C_{\mathbf{i j k}}$ and time $\mathbf{t}$. Notice that $\mathbf{p}=\mathbf{p}_{i j k}$ at cubic lattice $C_{i j k}$, and $\mathbf{p}_{i j k} \rightarrow \mathbf{p}^{*}$ as $h \rightarrow 0$ in the order of $o\left(h^{3}\right)$. Thus, from (4.5) and (4.6) to obtain the resultant error estimate as

$$
\left|J_{\lambda, i, j, k}\left(\mathbf{p}^{*}\right)-\hat{J}_{\lambda, i, j, k}\left(\mathbf{p}_{i j k}\right)\right| \leq \frac{2^{13}(6 !)^{4}}{13(12 !)^{3}} \mathbf{f}+4 c_{0} n^{\prime \prime} \mathbf{w}_{t} \mathbf{w}_{k} \mathbf{w}_{j} \mathbf{w}_{i} \epsilon
$$

where $\mathbf{f}=f^{(12)}\left(\theta_{x}, \theta_{y}, \theta_{z}, \tau\right), \mathbf{w}_{t}=\sum_{\mathbf{t}=1}^{6} w_{\mathbf{t}}, \mathbf{w}_{k}=\sum_{\mathbf{k}=1}^{6} w_{\mathbf{k}}, \mathbf{w}_{j}=\sum_{\mathbf{j}=1}^{6} w_{\mathbf{j}}, \mathbf{w}_{i}=\sum_{\mathbf{i}=1}^{6} w_{\mathbf{i}}$. That is $\hat{J}_{\lambda, i, j, k}\left(\mathbf{p}_{i j k}\right) \rightarrow J_{\lambda, i, j, k}\left(\mathbf{p}^{*}\right)$ in the order of $o\left(h^{12}\right)$ with the estimate $(4.7)$.

Consider the error estimate of $\mathbf{p}_{i j k} \rightarrow \mathbf{p}^{*}$ in the form of norm at cubic lattice $C_{i j k}$. Assume that there are exact coefficient pair $a_{i}^{*}(s), a_{i j}^{*}(s), a_{i j k}^{*}(s)$ for exact solution $\mathbf{p}^{*}(x, y, z, s)$. From (3.11) to suppose the exact coefficients are yielded that

$$
\begin{aligned}
& a_{i}^{*}(s)=\alpha_{i}^{*} s+\alpha_{0}^{*}, \quad i=1,2,3 . \\
& a_{i j}^{*}(s)=\beta_{i j}^{*} s^{2}+\beta_{i}^{*} s+\beta_{0}^{*}, \quad i=1,2,3 . j=1,2,3 . \\
& a_{i j k}^{*}(s)=\gamma_{i j k}^{*} s^{3}+\gamma_{i j}^{*} s^{2}+\gamma_{i}^{*} s+\gamma_{0}^{*}, \quad i=1,2,3 . j=1,2,3 . k=1,2,3 .
\end{aligned}
$$

By (3.11) and (4.8), there is $\epsilon_{1}>0$ to get estimate as

$$
\left\|a_{i}(s)-a_{i}^{*}(s)\right\|=\max _{s \in\left[s_{0}, \bar{s}\right]}\left|a_{i}(s)-a_{i}^{*}(s)\right| \leq\left(\alpha_{i}-\alpha_{i}^{*}\right) \bar{s}+\left(\alpha_{0}-\alpha_{0}^{*}\right) \leq \epsilon_{1} .
$$


As the argument in (4.11), there are $\epsilon_{2}, \epsilon_{3}>0$ for (4.9) and (4.10) such that

$$
\begin{aligned}
& \left\|a_{i j}(s)-a_{i j}^{*}(s)\right\| \leq\left(\beta_{i j}-\beta_{i j}^{*}\right) \bar{s}^{2}+\left(\beta_{i}-\beta_{i}^{*}\right) \bar{s}+\left(\beta_{0}-\beta_{0}^{*}\right) \leq \epsilon_{2}, \\
& \left\|a_{i j k}(s)-a_{i j k}^{*}(s)\right\| \leq\left(\gamma_{i j k}-\gamma_{i j k}^{*}\right) \bar{s}^{3}+\left(\gamma_{i j}-\gamma_{i j}^{*}\right) \bar{s}^{2}+\left(\gamma_{i}-\gamma_{i}^{*}\right) \bar{s}+\left(\gamma_{0}-\gamma_{0}^{*}\right) \leq \epsilon_{3} .
\end{aligned}
$$

Then, the estimate for the triply cubic polynomials is as follows,

$$
\begin{aligned}
& \left\|\mathbf{p}^{*}(x, y, z, s)-\mathbf{p}_{i j k}(x, y, z, s)\right\| \\
= & \max _{s \in\left[s_{0}, \bar{s}\right]} \max _{0 \leq x, y, z \leq L}\left|\mathbf{p}^{*}(x, y, z, s)-\mathbf{p}_{i j k}(x, y, z, s)\right| \\
\leq & {\left[a_{111}(s)-a_{111}^{*}(s)\right] \frac{\left(x-x_{i-1}\right)^{3}}{3 !}+\left[a_{222}(s)-a_{222}^{*}(s)\right] \frac{\left(y-y_{j-1}\right)^{3}}{3 !} } \\
& +\left[a_{333}(s)-a_{333}^{*}(s)\right] \frac{\left(z-z_{k-1}\right)^{3}}{3 !} \\
& +3\left[a_{112}(s)-a_{112}^{*}(s)\right] \frac{\left(x-x_{i-1}\right)^{2}}{2 !}\left(y-y_{j-1}\right)+3\left[a_{122}(s)-a_{122}^{*}(s)\right]\left(x-x_{i-1}\right) \frac{\left(y-y_{j-1}\right)^{2}}{2 !} \\
& +3\left[a_{113}(s)-a_{113}^{*}(s)\right] \frac{\left(x-x_{i-1}\right)^{2}}{2 !}\left(z-z_{k-1}\right)+3\left[a_{223}(s)-a_{223}^{*}(s)\right] \frac{\left(y-y_{j-1}\right)^{2}}{2 !}\left(z-z_{k-1}\right) \\
& +3\left[a_{133}(s)-a_{133}^{*}(s)\right]\left(x-x_{i-1}\right) \frac{\left(z-z_{k-1}\right)^{2}}{2 !}+3\left[a_{233}(s)-a_{233}^{*}(s)\right]\left(y-y_{j-1}\right) \frac{\left(z-z_{k-1}\right)^{2}}{2 !} \\
& +6\left[a_{123}(s)-a_{123}^{*}(s)\right]\left(x-x_{i-1}\right)\left(y-y_{j-1}\right)\left(z-z_{k-1}\right) \\
& +\left[a_{11}(s)-a_{11}^{*}(s)\right] \frac{\left(x-x_{i-1}\right)^{2}}{2 !}+\left[a_{22}(s)-a_{22}^{*}(s)\right] \frac{\left(y-y_{j-1}\right)^{2}}{2 !} \\
& \left.+\left[a_{33}(s)-a_{33}^{*}(s)\right] \frac{\left(z-z_{k-1}\right)^{2}}{2 !}+2\left[a_{12}^{*}\right)-a_{12}^{*}(s)\right]\left(x-x_{i-1}\right)\left(y-y_{j-1}\right) \\
& +2\left[a_{13}(s)-a_{13}^{*}(s)\right]\left(x-x_{i-1}\right)\left(z-z_{k-1}\right)+2\left[a_{23}(s)-a_{23}^{*}(s)\right]\left(y-y_{j-1}\right)\left(z-z_{k-1}\right) \\
& +\left[a_{1}(s)-a_{1}^{*}(s)\right]\left(x-x_{i-1}\right)+\left[a_{2}(s)-a_{2}^{*}(s)\right]\left(y-y_{j-1}\right)+\left[a_{3}(s)-a_{3}^{*}(s)\right]\left(z-z_{k-1}\right) \\
& +R_{s} \frac{\left(\bar{x}-x_{i-1}\right)^{4}+\left(\bar{y}-y_{j-1}\right)^{4}+\left(\bar{z}-z_{k-1}\right)^{4}}{4 !} \\
& +h e
\end{aligned}
$$

Use the estimate (4.11), (4.12) and (4.13), set $\bar{x}-x_{i-1}=\bar{y}-y_{j-1}=\bar{z}-z_{k-1}=h$, $\left(x-x_{i-1}\right)=\left(y-y_{j-1}\right)=\left(z-z_{k-1}\right)=h$, and $R_{s} \leq R_{0}$. Therefore

$$
\begin{aligned}
& \left\|\mathbf{p}^{*}(x, y, z, s)-\mathbf{p}_{i j k}(x, y, z, s)\right\| \\
\leq & 3 \epsilon_{3} \frac{h^{3}}{3 !}+24 \epsilon_{3} h^{3}+15 \epsilon_{2} \frac{h^{2}}{2 !}+6 \epsilon_{2} h^{2}+3 \epsilon_{1} h+R_{0} \frac{3 h^{4}}{4 !} .
\end{aligned}
$$

Clearly, $\mathbf{p}_{i j k}(x, y, z, s) \rightarrow \mathbf{p}^{*}(x, y, z, s)$ in the form of norm as $h \rightarrow 0$ at the order of $o\left(h^{3}\right)$ on each cubic lattice $C_{i j k}$ with the estimate (4.14).

Finally, from estimate errors (4.7) and (4.14) to find that for the total objective function (3.15), the convergence is in the order of $o\left(h^{3}\right)$ for constructed triply cubic polynomials $\mathbf{p}_{i j k}(x, y, z, s)$ approach, and in the order of $o\left(h^{12}\right)$ for performance index $J(\mathbf{p})$ at domain $\Omega$. 


\section{Computational procedure}

Render the computational procedure for the completeness of proposed triply cubic polynomials paradigm in the following steps:

Step 0 Given a partition number $N$ for domain $\Omega$.

Set $x_{1}=x, x_{2}=y, x_{3}=z$. Divide the cube domain $[0, L] \times[0, L] \times[0, L]$ into

$N \times N \times N$ cubic lattices $C_{i j k}$ with coordinates $x_{i}=\frac{i-1}{N}, y_{j}=\frac{j-1}{N}, z_{k}=\frac{k-1}{N}$ for $i=1,2, \ldots N, j=1,2, \ldots, N, k=1,2, \ldots, N$ at direction $x, y, z$.

- Define $\eta_{n}$ for $n=1,2, \ldots, \mathbf{n}$.

- Define sequences of triply cubic polynomials $p_{i j k}$ for each cubic lattice $C_{i j k}$.

- Define $\mathbf{p}_{i j k}^{\prime}, \mathbf{p}_{i j k}^{\prime \prime}$ at each cubic lattice $C_{i j k}$.

- Define $a_{i}(s), a_{i j}(s), a_{i j k}(s)$ at each cubic lattice $C_{i j k}$.

- Combine $\mathbf{p}_{i j k}(i, j, k, s)$ at each cubic lattice $C_{i j k}$.

- Take the base functions $\phi_{n}\left(x_{1}, x_{2}, x_{3}\right)$ for $n=1,2, \ldots, \mathbf{n}$ as given in (3.1).

Step 1 Execute iteration $\mathbf{p}_{i j k}(i, j, k, s)$ at each cubic lattice $C_{i j k}$.

Step 2 Compute $\nabla \mathbf{p}_{i j k}(i, j, k, s), \Delta \mathbf{p}_{i j k}(i, j, k, s)$ at each cubic lattice $C_{i j k}$. Set $\mathbf{L}\left(\mathbf{p}_{i j k}\right)$ as $L^{p}(t, i, j, k)$.

Step 3 Use $L^{p}(t, i, j, k)$ to calculate $\hat{J}_{\lambda, i, j, k}\left(\mathbf{p}_{i j k}\right)$ as Gauss quadrature approximate given at (3.17). Then, solve $a_{i}(s), a_{i j}(s), a_{i j k}(s)$ from $\hat{J}_{\lambda, i, j, k}\left(\mathbf{p}_{i j k}\right)$ for each cubic lattice $C_{i j k}$.

Step 4 Calculate $\nabla \eta(i, j, k, s), \Delta \eta(i, j, k, s)$ respectively, and combine $\phi_{k}\left(x_{1}, x_{2}, x_{3}\right)$ to get $q(i, j, k, s)$ to recover the unknown coefficient $\tilde{k}(x, y, z)$ at each lattice $C_{i j k}$.

Step 5 Summarizing of $C_{i j k}$ for $i, j, k$, get the total surface plot and contour plot of $\tilde{k}(x, y, z)$ at total domain $\Omega$.

Remark 5.1. For hyperbolic and elliptic cases, the triply cubic polynomial approach can be extended directly. (cf. $[6,7,8,9])$.

As well approach, experimental issue are acquired to evident the theoretic algorithm in our later works. Naturally, we omit it for the restrict of paper length. The task of this article is to address the new and novel point for constructing a triple cubic polynomial in finding the profile of unknown coefficient.

The detailed calculation issues (source data collections, redundant data elimination, domain partition, mesh expanding and recovery treatment) are exclusive in this paper.

\section{Conclusions}

As theoretical results, triple cubic polynomials approach for globally convergent algorithm is presented in this paper for solving the coefficient inverse problems in three 
dimensional case. It can be efficiently used to recover the unknown coefficients occurred in nonlinearity (cf. [19]). The theoretical issue subject to the parabolic initial-boundary equation is accomplished. Convergence proof and error estimate are provided for the proposed approach.

Upon with theoretic survey, the triple cubic polynomials approach supply a method to solve the wide applications in practical sense (cf. [20, 21]). This original investigation can be utilized to a variety of coefficient inverse problems as well as related areas.

The globally convergent algorithm consist of triple cubic polynomials approach exposit the possibility to achieve numerical approximation in high-dimensional case. As perspective of the future work, it would be a promise direction to tailor the triple cubic polynomials approach as a powerful and substantial methodology in theoretical research, laboratory experiments and industrial fields.

\section{Acknowledgments}

The author would like to thank the ICIP2010 and Journal of Physics: Conference Series for the article [3].

\section{References}

[1] Wang Q F 2005 Globally convergent algorithm for parabolic coefficient inverse problems Proceeding of Conference on Differential and Difference Equations and Applications 1109-1119

[2] Wang Q F 2006 Developed identication of coefficient inverse issue for 2D parabolic model by boundary pointwise observation International Conference Inverse Problems in Applied Sciences

[3] Wang Q F 2011 Bi-quadratic polynomials approach for globally convergent algorithm in high dimensions coefficient inverse problems Journal of Physics: Conference Series 290012015

[4] Klibanov M and Timonov A 2007 Numerical studies on the globally convergent convexification algorithm in 2D Inverse Problems 23 123-138

[5] Klibanov M and Timonov A 2007 A globally convergent convexification algorithm for multidimensional coefficient inverse problems Journal Inverse Ill-Posed Problems 15 167-179

[6] Klibanov M 1997 Global convexity in a three-dimensional inverse problem, SIAM J. Math. Anal. 28 1371-1388

[7] Klibanov M and Timonov A 2001 A new slant on the inverse problems of electromagnetic frequency sounding: convexification of a multiextremal objective function via the Carleman weight functions, Inverse Problems 17 1865-1887

[8] Klibanov M and Timonov A 2003 A globally convergent convexification algorithm for the inverse problem of electromagnetic frequency sounding in one dimension Numerical Methods and Programming 4 52-81

[9] Nachman A 1996 Global uniqueness for a two-dimensional inverse boundary value problem Ann. Math. 143 71-96

[10] Buhgeim A L and Klibanov M 1981 Globally uniqueness of a class of multimdimensional inverse problems Soviet Math. Dokl. 24 244-247

[11] Klibanov M and Timonov A 2004 A unified framework for constructing globally convergent algorithms for multidimensional coefficient inverse problems Applicable Analysis 83 993-955 
[12] Klibanov M and Timonov A 2003 A sequential minimization algorithm based on the convexification approach Inverse Problems 19 331-354

[13] Beilina L and Klibannov M 2010 Synthesis of global convergent and adaptivity for a hyperbolic coefficient inverse problems in 3D Journal of Inverse Ill-Posed Problems 18 85-132

[14] Beilina L and Klibanov M 2012 The Philosophy of the approximate global convergent for multidimensional coefficient inverse problems Complex Variables and Elliptic Equation 57277 299

[15] Shan H Klibanov M Liu H Pantong N and Su J 2008 Numerical implementation of the convexification algorithm for an optical diffusion tomograph Inverse Problem 24025006 1-18

[16] Beilina L and Klibanov M 2008 A globally convergent numerical method for a coefficient inverse problem, SIAM Journal on Scientific Computing 31 478-509

[17] Klibanov M 1992 Inverse problem and Carleman estimates, Inverse Problems 8 575-596

[18] Press W Teukolsky S Vetterling W and Flannery B 1988 Numerical Recipes in C (Cambridge University Press)

[19] Klibanov M and Timonov A 2004 Carleman Estimate for Coefficient Inverse Problems and Numerical Applications (Netherlands: VSP)

[20] Wang Q F 2011 Optimal Control for Nonlinear Parabolic Distributed Parameter Systems: with numerical analysis (Germany: Lambert Academic Publishing)

[21] Wang Q F 2011 Practical Application of Optimal Control Theory: computational approach (Germany: Lambert Academic Publishing) 